\title{
3D MAGNETIC RESONANCE IMAGING OF THE HUMAN BRAIN - NOVEL RADIAL SAMPLING, FILTERING AND RECONSTRUCTION
}

\author{
Maria Magnusson ${ }^{1,2,4}$, Olof Dahlqvist Leinhard ${ }^{2,4}$, Patrik Brynolfsson ${ }^{2,4}$, Per Thyr $^{2,4}$ and Peter Lundberg ${ }^{2,3,4,5}$ \\ Departments of ${ }^{1}$ Electrical Engineering, ${ }^{2}$ Radiation Physics, ${ }^{3}$ Radiology, and \\ ${ }^{4}$ Center for Medical Image Science and Visualization (CMIV), \\ Linköping University, and \\ ${ }^{5}$ Department of Radiation Physics, CKOC, University Hospital of Linköping, \\ SE-581 83 Linköping, Sweden \\ Email: maria@isy.liu.se, olof.dahlqvist.leinhard@liu.se, peter.lundberg@liu.se
}

\begin{abstract}
We have suggested a novel method PRESTO-CAN including radial sampling, filtering and reconstruction of $\mathrm{k}$-space data for 3D-plus-time resolved MRI. The angular increment of the profiles was based on the golden ratio, but the number of angular positions $\mathrm{N}$ was locked to be a prime number which guaranteed fix angle positions.

The time resolution increased dramatically when the profiles were partly removed from the k-space using the hourglass filter.

We aim for utilizing the MRI-data for fMRI, where the echo times are long, TE $\approx 37-40 \mathrm{~ms}$. This will result in field inhomogeneities and phase variations in the reconstructed images. Therefore, a new calibration and correction procedure was developed. We show that we are able to reconstruct images of the human brain with an image quality in line with what can be obtained by conventional Cartesian sampling.

The pulse sequence for PRESTO-CAN was implemented by modifying an existing PRESTO sequence for Cartesian sampling. The effort involved was relatively small and a great advantage will be that we are able to use standard procedures for speeding up data acquisition, i.e. parallel imaging with SENSE.
\end{abstract}

\section{KEY WORDS}

fMRI, MRI, radial reconstruction, golden ratio

\section{Introduction}

The neural activation of the brain in response to different stimuli such as visual stimulation, language tests, motor activities of hand and feet, etc, can be monitored using functional Magnetic Resonance Imaging (fMRI). The method is based on a change in the concentration ratio between oxygenated and de-oxygenated blood in the brain (blood oxygen dependent, or BOLD) in the specific area that is controlling the particular neural function. Such a change will affect the magnetic properties in the activated section of the brain. The examination method is often used for accurately delineating functional regions near brain tumours, thus fMRI has the potential to provide the surgeon with the information most needed at surgery, and it is considered to be an indispensable tool for predicting the extent of the functional impairment that might result as a function of the surgical procedures.

Two-dimensional (2D) Cartesian sampling using echoplanar imaging (2D EPI) is almost exclusively used for the acquisition of fMRI. However, there are several drawbacks with this sampling procedure, such as relatively low temporal and spatial resolution, as well as in-flow effects from adjacent 2D brain slices that are samples at different points in time. We have therefore explored the possibilities to combine Cartesian sampling with radial sampling of the MRI-data space, or 'k-space'. By using Cartesian sampling of the data in one dimension, it is possible to use a range of standard procedures for speeding up data acquisition in this domain. One such example is parallel imaging ('Sensitivity Encoding', or SENSE), but there are also other ways to optimize EPIdata sampling. This Cartesian sampling will then be merged with a modified radial sampling for obtaining a complete 3D k-space. We call this procedure PRESTOCAN, when the procedures are combined with the 'Principles of Echo Shifting using a Train of Observations' (PRESTO).

Radial sampling of k-space is known to simultaneously provide both high spatial and high temporal resolution. Recently, an optimal radial profile time order based on the Golden Ratio was presented in [1]. We have adopted and modified the idea, with a focus on higher temporal resolution without sacrificing any image quality. Also, since we aim for utilizing the data for fMRI, the echo times chosen for this application are long, TE $\approx 37-40 \mathrm{~ms}$. However; this will result in field inhomogeneities and phase variations in the reconstructed images. Some investigators move the position of the rotation axis when sampling radial data by removing phase from FFT(kspace). However, we cannot rely on this approach due to the phase variations in the reconstructed images. Therefore, a new calibration and correction procedure was devised. In this paper we show in simulations that the 
temporal resolution can be considerably improved. In addition, it is also demonstrated here that it is possible to produce images of a human brain that is essentially identical with that obtained using conventional Cartesian sampling.

The main aims with this work were to implement the new pulse sequences, to perform calibrations and corrections and finally to reconstruct the data both in simulated data, phantoms and in human subjects. In particular the temporal characteristics were investigated and presented here. Preliminary simulation results have previously been presented in [2] and [3].

\section{Materials and Methods}

\subsection{Sampling of k-space}

Three-dimensional (3D) sampling of k-space is normally performed using Cartesian sampling as in Fig. 1 left panel. In our method, PRESTO-CAN, the 3D k-space is sampled in the manner illustrated in Fig. 1 right panel, i.e. radial sampling in the $(\mathrm{kx}, \mathrm{kz})$-plane and Cartesian sampling in the ky-direction. In the following we concentrate on the radial data, which is equivalent to $2 \mathrm{D}$ data space, but it can very easily be extended to 3D. Moreover, Fig. 1 illustrates that every profile in k-space is sampled with one excitation only, i.e. EPI=1 or singleshot. In the experiments we used higher EPI-factors.
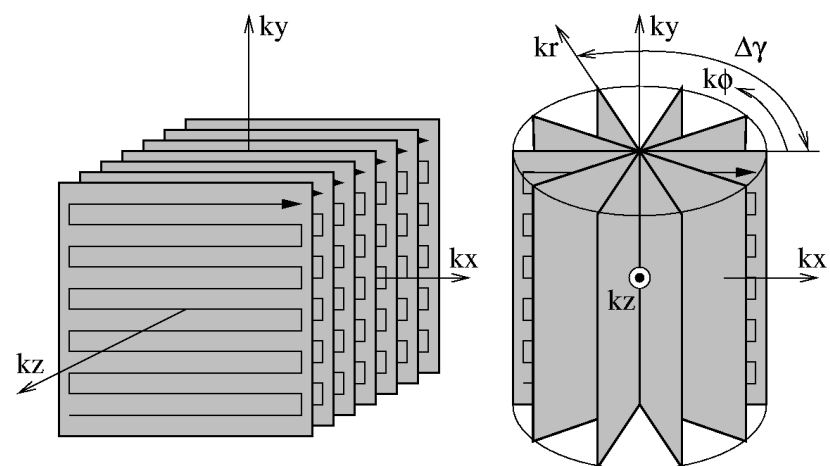

Figure 1 Left: Cartesian sampling. Right: Our new radial PRESTO-CAN sampling pattern.

In [1], the k-space was sampled radially using the angular increment $\Delta \gamma_{\text {orig }}$ of the radial profiles,

$$
\Delta \gamma_{\text {orig }}=180^{\circ} \frac{2}{\sqrt{5}+1} \approx 111^{\circ}
$$

i.e. $\Delta \gamma_{\text {orig }}$ was chosen to be $180^{\circ}$ divided by the 'golden ratio'.

This guarantees that if an arbitrary time interval is chosen, the profiles within that interval will be spread out almost evenly across k-space. Note however, that since the golden ratio is an irrational number, the same angular position will never be visited again. This might be a disadvantage; therefore have modified the idea as follows.

We chose to sample $\mathrm{k}$-space with $\mathrm{N}$ radial profiles at $\mathrm{N}$ fixed angle positions. Let the profile numbers be $n=0,1$,
$2, \ldots, \mathrm{N}-1$ with the corresponding angles $\mathrm{n} 360^{\circ} / \mathrm{N}$. The smallest angular difference between profiles will then be $\Delta \phi=360^{\circ} /\left(2^{*} \mathrm{~N}\right)$. Among the angles, the one which is closest to $\Delta \gamma_{\text {orig }}$ was chosen, and it has the profile number $\mathrm{n}=\mathrm{M}$, i.e. the angular increment is chosen to be

$$
\Delta \gamma=360^{\circ} \mathrm{M} / \mathrm{N} \approx \Delta \gamma_{\text {orig }}=180^{\circ} \frac{2}{\sqrt{5}+1} \approx 111^{\circ}
$$

It is important that all profiles are visited before repeating the first profile. This is guaranteed if the number of profiles is chosen to be a prime number, [4]. The eligible number of profiles can be further extended by also considering cases when $\mathrm{M}$ and $\mathrm{N}$ have no common factors, for example $\mathrm{N}=10$ and $\mathrm{M}=3$ [5].

The sampling pattern is illustrated in Fig. 2, where the number of profiles $N=7$. The profile numbers are $\mathrm{n}=0,1,2,3,4,5,6$ with the corresponding angles $\mathrm{n} 360^{\circ} / 7$. The angular increment is $\Delta \gamma=360^{\circ} \mathrm{M} / \mathrm{N} \approx 360^{\circ} 2 / 7 \approx 103^{\circ}$, where the profile number $M=2$. The smallest angular difference between profiles are $\Delta \phi=360^{\circ} /(2 \cdot 7) \approx 26^{\circ}$.

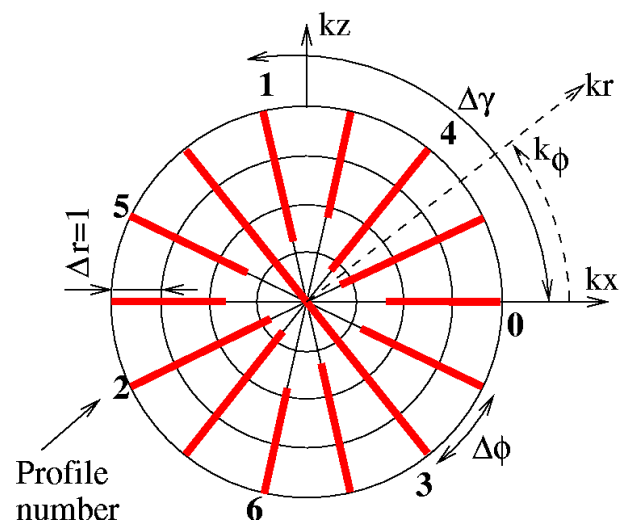

Figure 2 PRESTO-CAN sampling of $k$-space.

\subsection{Hourglass Filtering}

In Fig. 3, the profiles from Fig. 2 were plotted with respect to time. The profile numbers $\mathrm{n}=0,1,2,3,4,5,6$ agree with the time order $\mathrm{t}=0,1,2,3,4,5,6,0,1,2,3, \ldots$

See Fig. 2. To avoid under-sampling, the angular sampling density $r \pi / \mathrm{N}$ should not exceed 1, i.e. $\mathrm{r} \leq \mathrm{N} / \pi$. For $\mathrm{N}=7, \mathrm{r}=7 / \pi=2.23$. For a radius larger than 2.23 , aliasing will appear as streaks in the image. However, a small portion of aliasing can be tolerated; in particular as a smaller number of profiles will provide a better time resolution. On the other hand, for $r=0$ only one profile is required. If the points fulfilling $r=N / \pi$ are connected, an hourglass-shaped contour is obtained. The points outside the hourglass are not needed and can therefore be removed thereby providing much better time resolution. Note that the current time point of interest in Fig. 3 is $t=3$, and that profiles collected both previously $(t<3)$ and in the future $(t>3)$ are needed. The hourglass filter is consequently a non-causal filter. However, this is not a problem since the profiles can be recorded and reconstructed later. The hourglass filter will move 
forward in time as indicated by the arrow. For each time position a new volume will be reconstructed.

There is a possibility that the rapid change in sampling density where the hourglass cuts the profile introduces artifacts in the reconstruction later. This was avoided by using angular interpolation in which the removed data points were linearly interpolated from the neighbors in the angular direction, see Fig. 4. This is a simple way to treat the pre-compensation step in section 2.3 step 1). An alternative method is to skip the angular interpolation and to use the method described by Johnson and Pipe [6] instead.

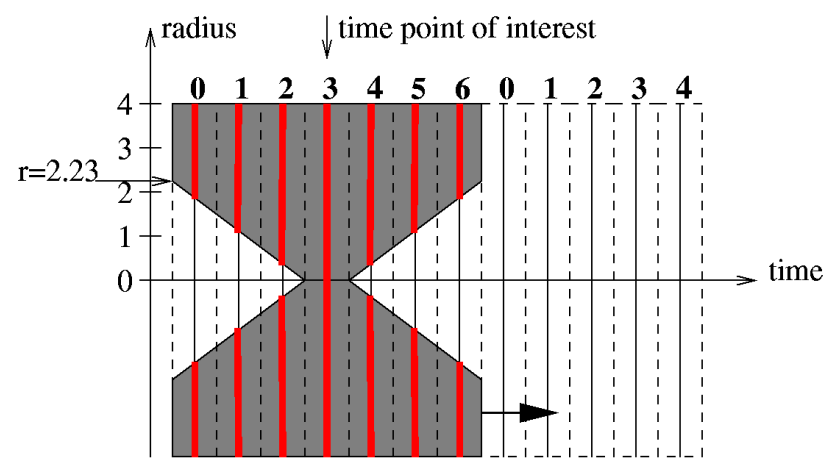

Figure 3 The profiles plotted against time. The moving hourglass filter.

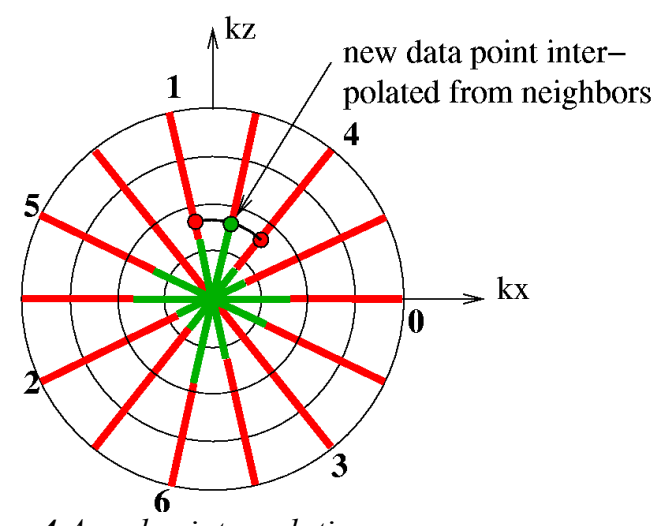

Figure 4 Angular interpolation.

\subsection{Reconstruction Using Gridding}

The previously reported 2D gridding method [7], [8], was extended to our 3D geometry in Fig. 1 and used for image reconstruction. The algorithm is illustrated in Fig. 5 and consists of the following steps:

1. Pre-compensation with the local sampling density. The pre-compensation was proportional to $2 \pi \mathrm{r} / \mathrm{N}$ except for the samples at the origin which was compensated using $0.52 \pi / \mathrm{N}$. The data points are over-sampled a factor of two in the radial direction. This is common in the frequency encoding direction.

2. Resampling with forward mapping using the interpolation function $C(k x, k z)=C(k x) \cdot C(k z)$, where
$C()$ is a 4-point Kaiser-Bessel filter. The same interpolation function was used for both data values and weight values. The sampling distance of the Cartesian grid $\Delta \mathrm{x}$ was chosen to the two times oversampled radial sampling distance, i.e. $\Delta \mathrm{r}=1 / 2$.

3. Post-compensation using the weight function, $\mathrm{W}(\mathrm{kx}, \mathrm{kz})$. Since pre-compensation was performed in step 1), the post-compensation had only a marginal effect.

4. 3D inverse Fourier transform.

5. Division with $\mathrm{c}(\mathrm{x}, \mathrm{y})$, the inverse Fourier transform of $\mathrm{C}(\mathrm{kx}, \mathrm{ky})$ and removing outer data points due to the over-sampling factor two.

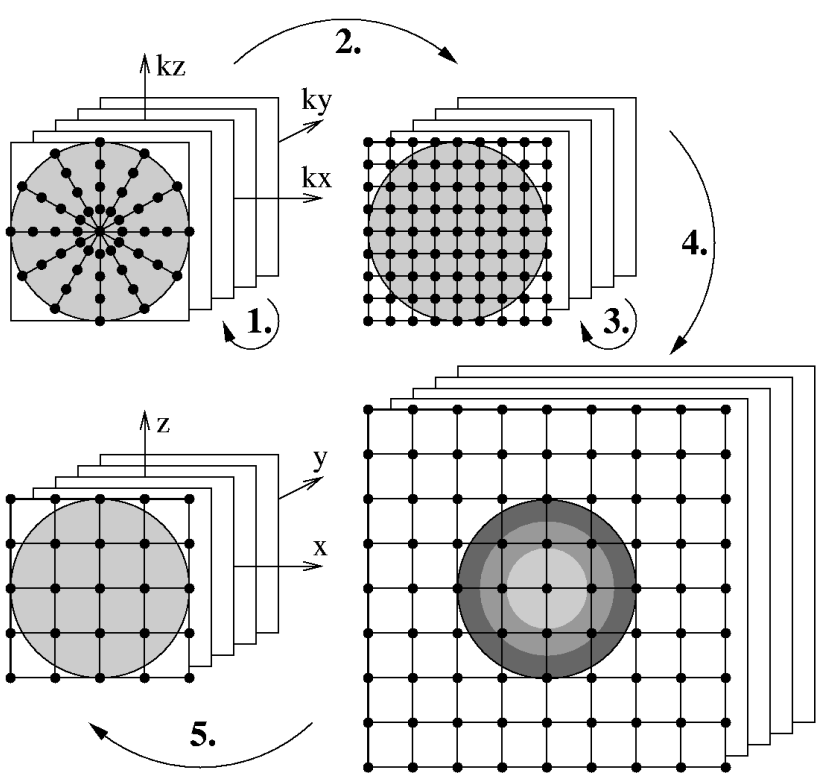

Figure 5 The $2 D$ gridding method extended to our $3 D$ geometry.

\subsection{Magnetic Resonance Imaging}

All data from human subjects and phantoms were acquired using a $1.5 \mathrm{~T}$ Achieva MR-scanner (Philips Medical Systems, The Netherlands). An 8-channel head coil was used for data acquisition. Agar phantoms were manufactured using a suitably bottle shaped container of non-magnetic material.

\section{Results}

\subsection{Time-resolved Simulated Data}

Two properties of PRESTO-CAN were investigated in this experiment, the temporal resolution and image quality. The large elliptical object with smaller objects inside did not move. It was primarily used for checking the image quality. The small circular object on the left was removed instantaneously in the middle of the time interval and it was used for evaluating the temporal step response. Fig. 6 left shows the reconstructed image using the conventional sliding window i.e. no hourglass was applied, and Fig. 6 middle panel shows the influence of 
hourglass with angular interpolation. The image size was $128 \times 128$ and the number of profiles was $\mathrm{N}=199$. The width of the hourglass was 1 profile at the center $(r=0)$ and it reached full width at $r=54$. In this simulation experiment, we did not use over-sampling with two as described in section 2.3, step 1 and 2. Fig. 6 right shows how the intensity of the removed circular left outer object changes with time. The temporal resolution was defined as the transition time from $80 \%$ to $20 \%$ intensity. Note how the temporal resolution was increased by a factor of 6.5 for hourglass and angular interpolation. At the same time, the image quality was almost intact and stable over time.
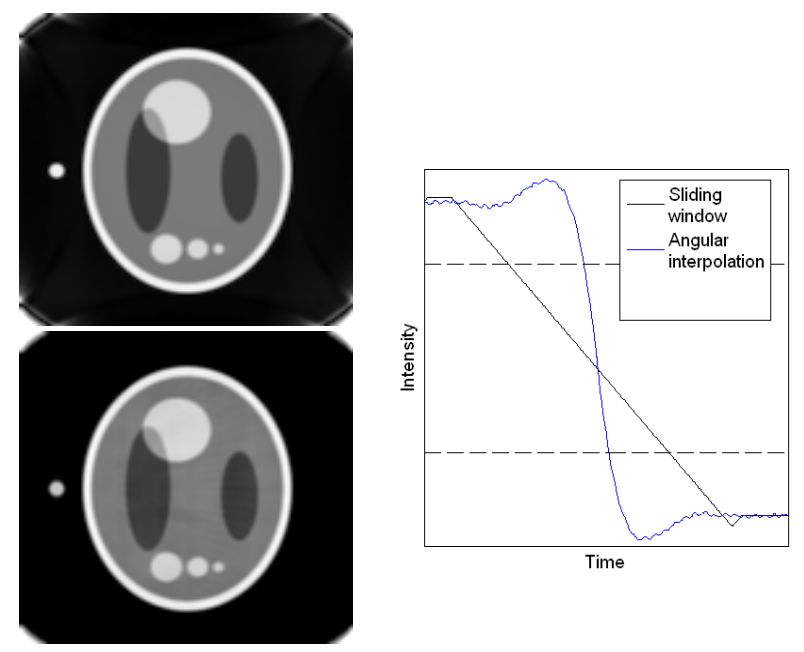

Figure 6 Upper left: Conventional sliding window. Lower left: Hourglass filtering. Right: Temporal resolution for the small left object.

\subsection{Human Brain Images}

In this section the image quality that can be obtained using the new (3D) PRESTO CAN-sampling pattern is described. If one compare it with the Cartesian sampled data in Figure 1, left, it is obvious that the same image quality can be obtained. As the data acquisition is aimed for $\mathrm{fMRI}$, the echo times chosen were long, $\mathrm{TE} \approx 37-40$ ms. This resulted in field inhomogenities and phase variations in the reconstructed images, see Fig. 13 and 14. Some authors, see for example [9] and [10], move the rotation axis in position by removing phase from FFT(kspace). This approach could not be used here due to the phase variations in the reconstructed images. Therefore, a new calibration and correction procedure had to be developed.

The experiments were performed on a Philips 1.5T Achieva scanner using a PRESTO pulse sequence that performed Cartesian sampling of k-space (as shown in Fig. 1 left panel). The pulse sequence was also modified to radial PRESTO CAN sampling, shown in Fig. 1, right panel.

Measurements on a phantom filled with agar and on the brain of a volunteer were performed. The repetition time
(TR) and echo time (TE) was TR/TE=22/38 for the Agar phantom and $\mathrm{TR} / \mathrm{TE}=24 / 40$ for the brain. The EPI-factor was $\mathrm{EPI}=3$ for the agar bottle and $\mathrm{EPI}=15$ for the brain. To illustrate the calibration/correction procedure below we chose to use the agar data.

For the Cartesian sampling, k-space was sampled at $(\mathrm{kx}, \mathrm{ky}, \mathrm{kz})=(160,75,41)$ points and reconstructed to a $(\mathrm{x}, \mathrm{y}, \mathrm{z})=(80,80,41)$ volume. For the radial sampling, $\mathrm{k}-$ space was sampled at $(\mathrm{kr}, \mathrm{ky}, \mathrm{k} \phi)=(160,75,79)$ points $((160,78,79)$ if $\mathrm{EPI}=3)$ and reconstructed to a $(\mathrm{x}, \mathrm{y}, \mathrm{z})=(80,80,80)$ volume which was later truncated to a $(\mathrm{x}, \mathrm{y}, \mathrm{z})=(80,80,41)$ volume so that it could easily be compared with the Cartesian sampling.

\subsubsection{Corrections of Real Data}

Unlike simulated data, real data must be corrected in a number of ways prior to reconstruction. To achieve this, a reference scan kref of k-space needs to be acquired and used for calibration. The reference scan used here was similar to a normal full scan of $\mathrm{k}$-space, the only difference being that the ky-gradient was not used, i.e. $\mathrm{ky}=0$.

The full calibration procedure of the reference scan was performed as follows:

1. Compute forward-and-back correction vectors for every profile, i.e. every $\mathrm{k} \phi$-angle in the reference scan.

2. Apply a linear phase correction $\mathrm{k} \cdot \mathrm{n}$, where $\mathrm{n}$ is the profile number, and also the golden angles to consecutive angles. Interpolate missing angles so that for every angle $\mathrm{k} \phi$ there exist an angle $\mathrm{k} \phi+180^{\circ}$. Locate rotation axis position for every $\left(\mathrm{k} \phi, \mathrm{k} \phi+180^{\circ}\right)$ profile pair in the reference scan by using complex correlation to obtain the rotation-angle-position.

3. Determine the phase drift at the rotation axis position for every $\mathrm{k} \phi$ profile in the reference scan and produce a phase-drift correction vector.

The subsequent full correction procedure of $k$-space data was performed as follows:

4. Perform forward-and-back correction, using the forward-and-back correction vectors obtained in the calibration procedure, for every single profile and $\mathrm{k} \phi$ angle in k-space.

5. Perform rotation-angle-position corrections.

6. Perform phase-drift corrections.

In the figures below the calibration procedure is presented and also the correction procedure applied on the reference data from the agar bottle. (Correction is normally not performed on reference data, only the normal k-space data.)

In step 1, the correction vectors are calculated as the phase difference between right and left rows of the 1D 
Fourier transform in the radial direction of the reference scan. More specifically, let $\operatorname{ref}(\mathrm{r}, \mathrm{ky})=\mathrm{FFT}_{\mathrm{r}}(\mathrm{kref}(\mathrm{kr}, \mathrm{ky})$, then the forward-and back correction vector can be calculated as

$$
\arg ((\operatorname{ref}(\mathrm{r}, \mathrm{ky}-\mathrm{A})+\operatorname{ref}(\mathrm{r}, \mathrm{ky}+\mathrm{A})) / \operatorname{ref}(\mathrm{r}, \mathrm{ky})) ;
$$

where ky is a 'left scan row', ky-A the preceding 'right row' and ky $+\mathrm{A}$ the subsequent 'right row'. For better accuracy, all left scan rows can be averaged. Thus there will be one vector for each profile angle $\mathrm{k} \phi$. The forwardand-back correction vector that was obtained for $\mathrm{k} \phi=0^{\circ}$ is shown in Fig. 7. Note that a straight line essentially corresponded to a translation in k-space. The "noisy" data points (less than -5 and above +27 ) will not be a problem since they correspond to background voxels outside the object.

During the correction step 4, the 1D Fourier transform in the radial direction will be applied to k-space data and the phase of all left rows will be adjusted by the forward-andback correction vector.

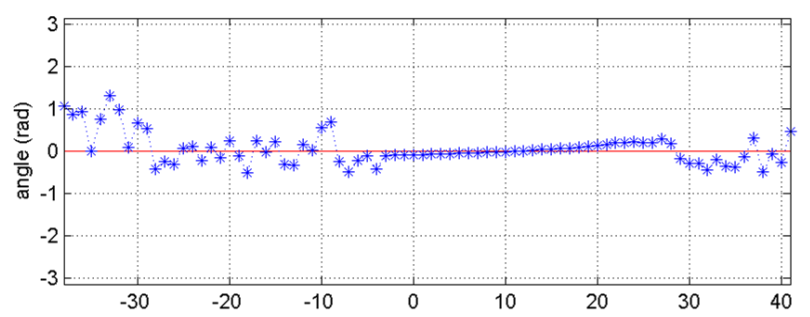

Figure 7 The forward-and-back correction vector.
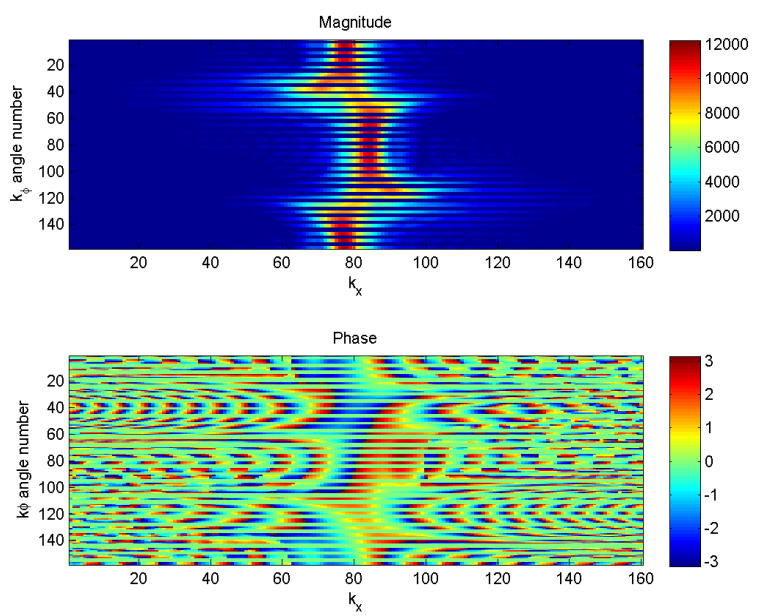

Figure 8 Re-sorted reference data.

In step 2, we choose one ky-position in kref and re-sorted the golden angles to consecutive angles in the interval [0$360^{\circ}$ ]. This gave a $2 \mathrm{D}$-matrix $\operatorname{kref}(\mathrm{kr}, \mathrm{k} \phi)$, see Fig. 8 . The missing $\mathrm{k} \phi$ angles are indicated with rows equal to zero. We also applied a linear phase correction $k \cdot n$, where $n$ is the profile number. The factor $\mathrm{k}$ should be chosen such that the phases obtained for $\mathrm{k} \phi=0^{\circ}$ and $\mathrm{k} \phi=360^{\circ}$ overlap. Also, the phase should vary as slowly as possible between consecutive angles. This is shown in Fig. 8. Then the empty rows where filled with data obtained using linear interpolation in magnitude and phase separately, see Fig. 9. The rotation axis position were obtained by complex correlation for every $\left(\mathrm{k} \phi, \mathrm{k} \phi+180^{\circ}\right)$ profile pair in $\mathrm{kref}(\mathrm{kr}, \mathrm{k} \phi)$. A line was adjusted to the correlation result and the rotation-angle-position was then determined, in the present case it was 0.25 offset from the central position $\mathrm{kr}=81$.
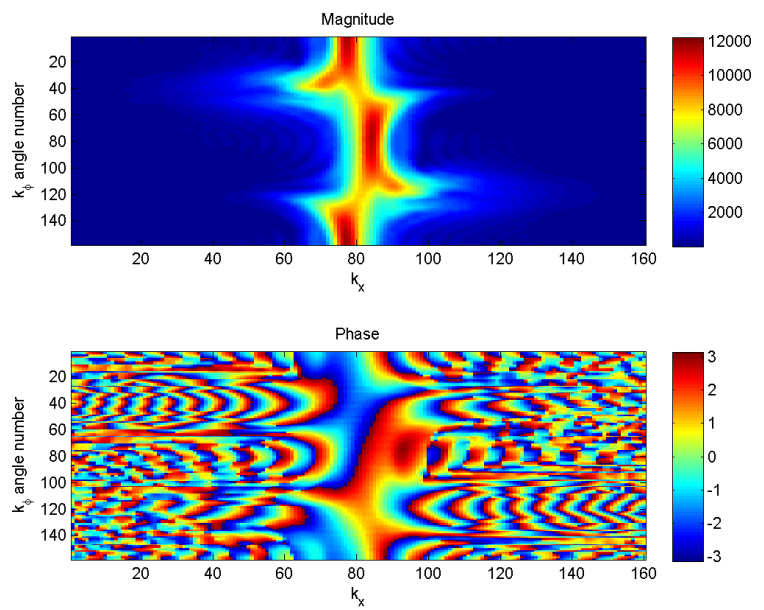

Figure 9 Interpolated reference data.
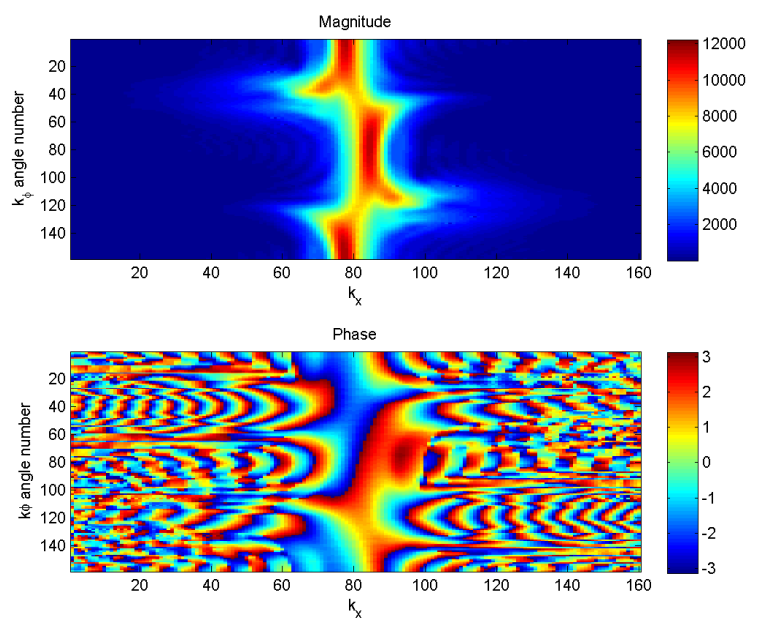

Figure 10 Rotation axis adjusted reference data.

During the correction step 4, k-space data is translated such that the rotation angle position will be located at position $\mathrm{kr}=81$. After applying this correction to $\mathrm{kref}(\mathrm{kr}, \mathrm{k} \phi)$, the result will be as is shown in Fig. 10. Note that it appears essentially as Fig. 9 since the offset was only 0.25 .

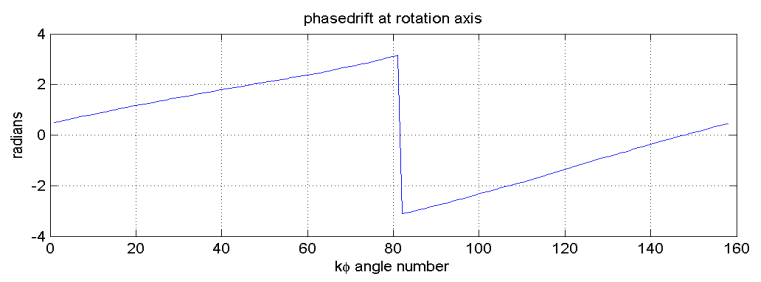

Figure 11 Phase-drift vector.

In step 3, the phase drift at the rotation axis position was computed. Note that the phase at $\mathrm{kr}=81$ in Fig. 10 is varying in the whole phase range $[-\pi, \pi]$. This must be 
adjusted since the rotation axis position $\mathrm{kr}=81$ denote the same position in $\mathrm{k}$-space. The phase variation at $\mathrm{kr}=81$ multiplied with -1 gives a phase-drift correction vector, see Fig. 11.
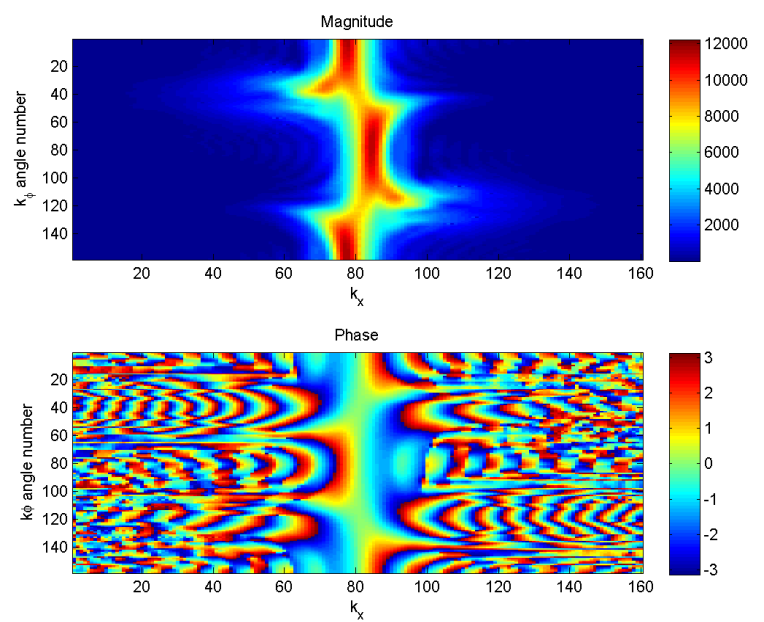

Figure 12 Phase-drift corrected reference data.

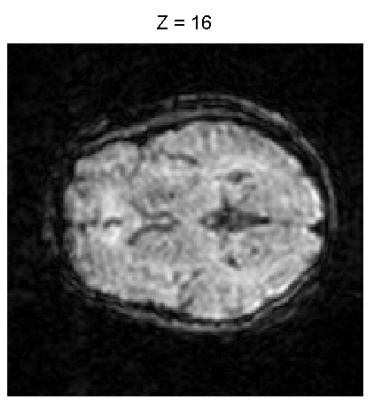

$z=16$

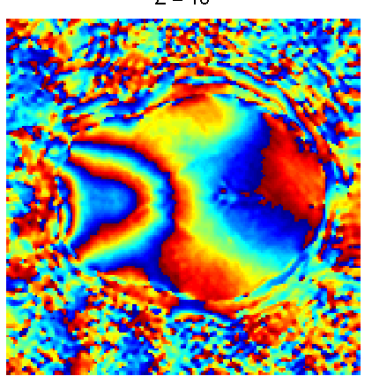

$\mathrm{X}=34$

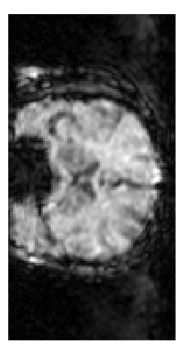

$X=54$

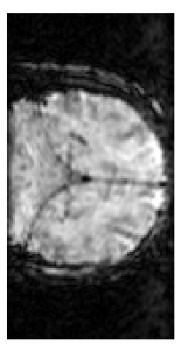

$Z=21$

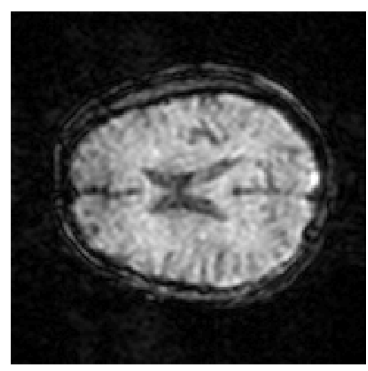

$Z=21$

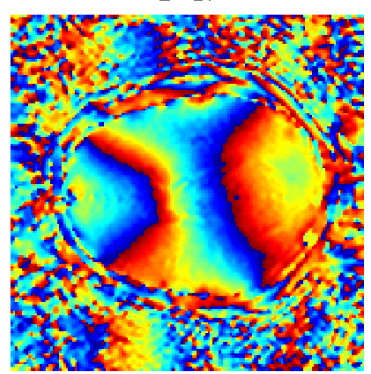

$Y=41$

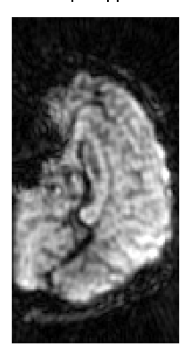

$Y=51$

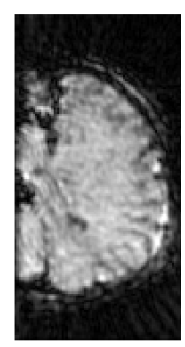

Figure 13 Reconstructed images from radially sampled PRESTO-CAN data. Both magnitude and phase images are shown.

During the correction step 6, the phase of the k-space data will be adjusted by adding the phase of the phase-drift correction vector. When applying this correction to the rotation axis, the corrected version of $\mathrm{kref}(\mathrm{kr}, \mathrm{k} \phi)$, the result is as shown in Fig. 12.

\subsubsection{Reconstructing Real Data}

When the real data has been corrected, it can be reconstructed in a similar manner as simulated data. Here we show two different reconstructed volumes of the human brain suitable for fMRI. Volume 1 was reconstructed from radially sampled data and volume 2 was reconstructed from Cartesian sampled data.

The radially sampled data were subjected to the whole calibration and correction procedure described in the previous section. The Cartesian sampled data were instead subjected to a forward-and-back correction provided by the Philips MR-scanner similar to the correction described in the previous section.

In a final step, the reconstructed volumes were zeropadded once in the Fourier domain.

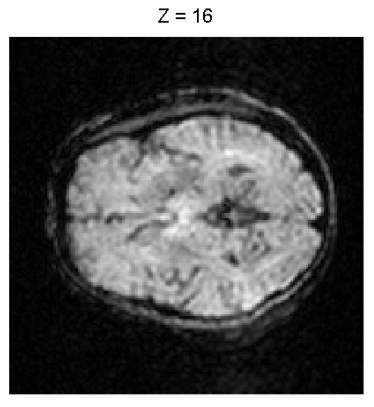

$Z=16$

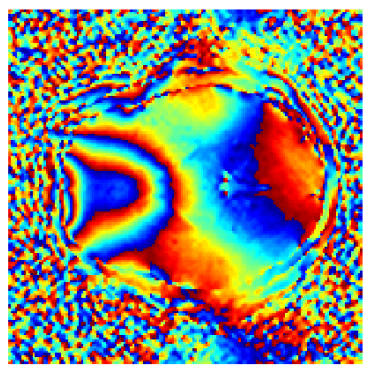

$\mathrm{X}=34$

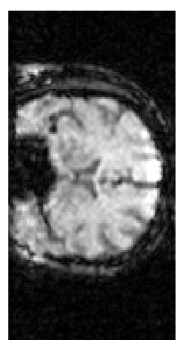

$\mathrm{X}=54$

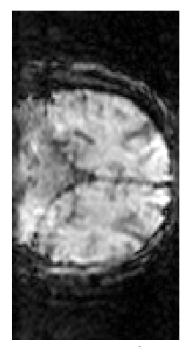

$z=21$

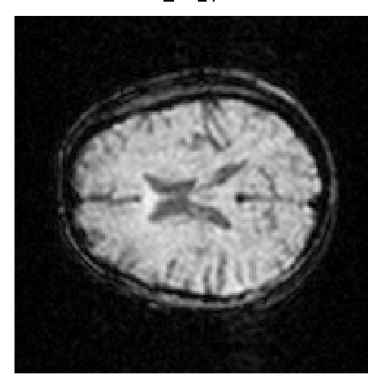

$Z=21$

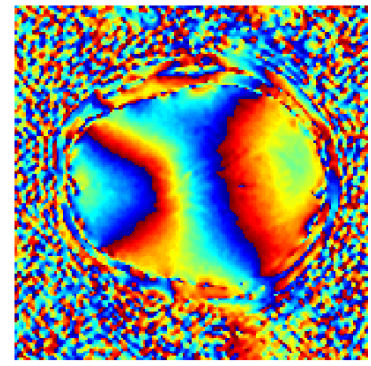

$Y=41$

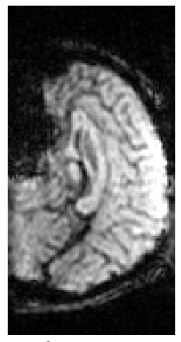

Figure 14 Reconstructed images from Cartesian sampled PRESTO data. Both magnitude and phase images are shown.

Volume 1, reconstructed from radially sampled data is shown in Fig. 13, and volume 2 reconstructed from Cartesian sampled data is shown in Fig. 14. The figures show different axial slices, and the corresponding phase 
images are also shown. Note the close similarity in both magnitude and phase between Fig. 13 and 14.

In Fig. 15, the hourglass filtration and subsequent angular interpolation were applied before reconstruction. Note that image quality is virtually identical in Fig. 13 and Fig. 15. An hourglass applied at another time point would result in a similar result. This means that the image is stable over time. The angular interpolation can also be replaced with the alternative pre-compensation method described by Johnson and Pipe [6].

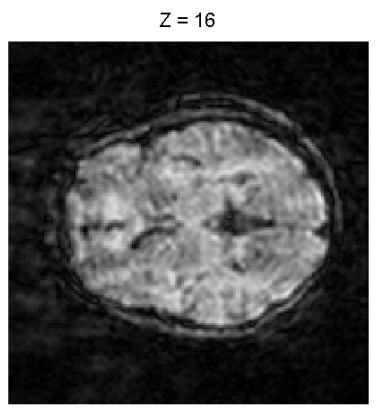

$Z=16$

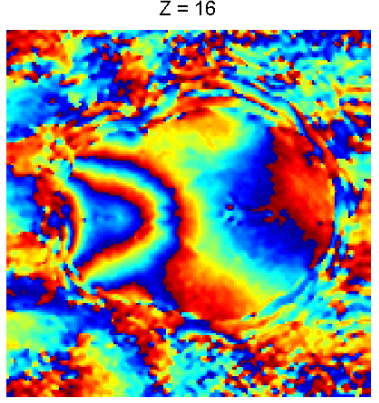

$x=34$

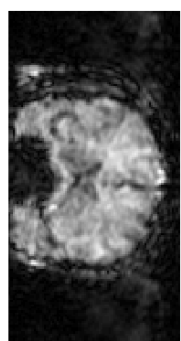

$X=54$

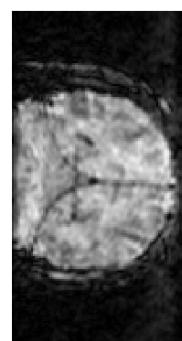

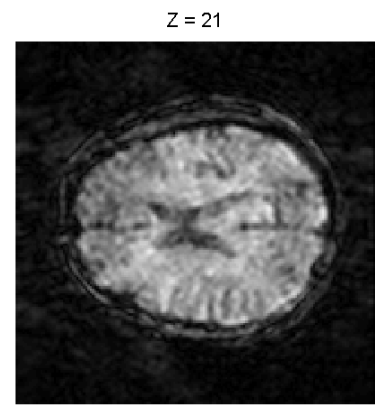

$Z=21$

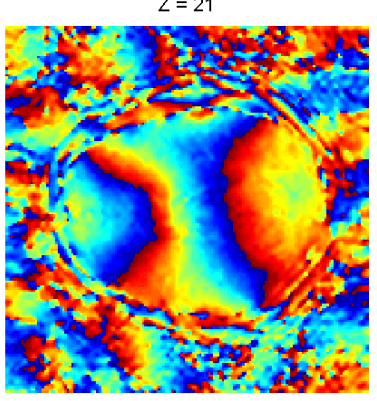

$Y=41$

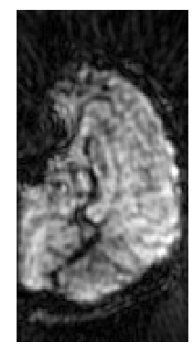

$Y=51$

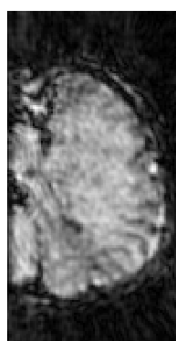

Figure 15 Reconstructed images from radially sampled PRESTO-CAN data. Hourglass filtration and angular interpolation were applied prior to reconstruction. Both magnitude and phase images are shown.

\section{Discussion and Conclusion}

We have proposed and implemented a novel method (PRESTO-CAN) that included radial sampling, filtering and reconstruction of k-space data for dynamically resolved 3D MRI (3D plus time). The angular increments of the profiles were based on the golden ratio, similar to [1]. In [1] however, the angular increment was based on an irrational number which can lead to time varying artefacts even when conventional sliding window technique is used. We therefore locked the number of angular positions $\mathrm{N}$ to be a prime number which guaranteed fix angle positions.

The time resolution increased dramatically when the profiles were partially removed from the $\mathrm{k}$-space using the hourglass filter.

Since, we primarily aim for using the procedures for fMRI, the echo times chosen for this application are long, $\mathrm{TE} \approx 37-40 \mathrm{~ms}$. This will result in field inhomogeneities and phase variations in the reconstructed images. Therefore, a new calibration and correction procedure had to be developed. We have shown that it is possible to reconstruct images of the human brain obtained using PRESTO-CAN and hour-glass filtering with an image quality that is at least equivalent to what can be obtained by conventional Cartesian sampling. However PRESTOCAN is much more efficient than Cartesian sampling and both the spatial and temporal resolutions can be expanded far beyond what is possible using the inefficient Cartesian dynamic sampling pattern. The image distortion can also be reduced in the process, although this remains to be shown. In fMRI all these three aspects are very important as neurological activation patterns are intrinsically rapid events that occurs on a spatially resolved scale. In conventional fMRI the effects of image distortion are often a significant problem.

Finally, the pulse sequence for PRESTO-CAN was obtained by modifying a conventional PRESTO sequence for Cartesian sampling. A great advantage with PRESTOCAN is therefore that it is possible to use standard procedures for speeding up data acquisition; one such is parallel imaging (SENSE etc).

\section{Acknowledgements}

Financial support from the Swedish National Research Council (VR), the Cancer Foundation (CF), the Knowledge foundation (KK), and the University Research Foundations are gratefully acknowledged. We also would like to acknowledge skillful contributions to various parts of this project by Eva Leuchovius, Marcel Warntjes, and Anders Tisell.

\section{References}

[1] S. Winkelmann, T. Schaeffter, T. Koehler, H. Eggers, \& O. Doessel, An optimal radial profile order based on the golden ratio for time-resolved MRI, IEEE Trans. Med. Imaging, Vol. 26, No.1, 2007, 68-76.

[2] M. Magnusson, O. Dahlqvist Leinhard, P. Brynolfsson, P. Lundberg, Radial k-space sampling: step response using different filtering techniques, ISMRM Workshop on Data sampling and Image Reconstruction, The Enchantment Resort, Sedona, Arizona, USA, January 25-28, 2009 
[3] M. Magnusson, O. Dahlqvist Leinhard, P. Brynolfsson, P. Lundberg, Improved temporal resolution in radial k-space sampling using an hourglass filter, Proceedings of the ISMRM 17th Scientific Meeting \& Exhibition, Honolulu, Hawaii, USA, April 18-24, 2009

[4] J. Sunnegårdh, Combining analytical and iterative reconstruction in helical cone-beam CT, Linköping Studies in Science and Technology, Thesis No. 1301, 2007.

[5] Personal communication with Klas Nordberg at Dept. of Electrical Engineering, Linköping University, Sweden.

[6] K. O. Johnson, J. Pipe, Convolution kernel design and efficient algorithm for sampling density correction, Magnetic Resonance in Medicine, Vol. 61, 2009, 439-447.

[7] J. O'Sullivan, A fast sinc function gridding algorithm for Fourier inversion i computer tomography, IEEE Trans. Med. Imaging, Vol. MI-4, No.4, 1985, 200-207.

[8] Pauly: Non-Cartesian Reconstruction, Lecture notes, URL:http://www.stanford.edu/class/ee369c/notes/non_car t_rec_07.pdf, 2007

[9] J. Pipe, Motion correction with PROPELLER MRI: Application to head motion and free-breathing cardiac imaging, Magnetic Resonance in Medicine, Vol. 42, 1999, 963-969.

[10] J. Pipe, N. Zwart, Turboprop: Improved PROPELLER imaging, Magnetic Resonance in Medicine, Vol. 55, 2006, 380-385. 